\title{
Dental characters used in phylogenetic analyses of mammals show higher rates of evolution, but not reduced independence
}

\author{
Neil Brocklehurst ${ }^{\text {Corresp., } 1}{ }^{,}$Gemma L Benevento ${ }^{2}$ \\ 1 Department of Earth Sciences, University of Oxford, Oxford, United Kingdom \\ 2 School of Geography, Earth and Environmental Sciences, University of Birmingham, Birmingham, United Kingdom \\ Corresponding Author: Neil Brocklehurst \\ Email address: neil.brocklehurst@earth.ox.ac.uk
}

Accurate reconstructions of phylogeny are essential for studying the evolution of a clade, and morphological characters are necessarily used for the reconstruction of the relationships of fossil organism. However, variation in their evolutionary modes (for example rate variation and character non-independence) not accounted for in analyses may be leading to unreliable phylogenies. A recent study suggested that phylogenetic analyses of mammals may be suffering from a dominance of dental characters, which were shown to have lower phylogenetic signal than osteological characters and produced phylogenies less congruent with molecularly-derived benchmarks. Here we build on this previous work by testing five additional morphological partitions for phylogenetic signal and examining what aspects of dental and other character evolution may be affecting this, by fitting models of discrete character evolution to phylogenies inferred and time calibrated using molecular data. Results indicate that the phylogenetic signal of discrete characters correlate most strongly with rates of evolution, with increased rates driving increased homoplasy. In a dataset covering all Mammalia, dental characters have higher rates of evolution than other partitions. They do not, however, fit a model of independent character evolution any worse than other regions. Primates and marsupials show different patterns to other mammal clades, with dental characters evolving at slower rates and being more heavily integrated (less independent). While the dominance of dental characters in analyses of mammals could be leading to inaccurate phylogenies, the issue is not unique to dental characters and the results are not consistent across datasets. Molecular benchmarks (being entirely independent of the character data) provide a framework for examining each dataset individually to assess the evolution of the characters used. 
2

3 Dental Characters Used in Phylogenetic Analyses of Mammals Show Higher Rates of Evolution,

4

5

6

7

8

$9{ }^{1}$ Department of Earth Sciences, University of Oxford, South Parks Road, Oxford, UK, OXI 3QR,

10 neil.brocklehurst@earth.ox.ac.uk

112 School of Geography, Earth and Environmental Sciences, University of Birmingham,

12 Birmingham, UK, B152TT,g.l.benevento@bham.ac.uk

13

14

15

16

17

18

19

20

21

22

23 
ABSTRACT

Accurate reconstructions of phylogeny are essential for studying the evolution of a clade, and morphological characters are necessarily used for the reconstruction of the relationships of fossil organism. However, variation in their evolutionary modes (for example rate variation and character non-independence) not accounted for in analyses may be leading to unreliable

31 phylogenies. A recent study suggested that phylogenetic analyses of mammals may be suffering

32 from a dominance of dental characters, which were shown to have lower phylogenetic signal

than osteological characters and produced phylogenies less congruent with molecularly-derived

34 benchmarks. Here we build on this previous work by testing five additional morphological partitions for phylogenetic signal and examining what aspects of dental and other character evolution may be affecting this, by fitting models of discrete character evolution to phylogenies inferred and time calibrated using molecular data. Results indicate that the phylogenetic signal of discrete characters correlate most strongly with rates of evolution, with increased rates driving increased homoplasy. In a dataset covering all Mammalia, dental characters have higher rates of evolution than other partitions. They do not, however, fit a model of independent character evolution any worse than other regions. Primates and marsupials show different patterns to other mammal clades, with dental characters evolving at slower rates and being more heavily

43 integrated (less independent). While the dominance of dental characters in analyses of mammals 44 could be leading to inaccurate phylogenies, the issue is not unique to dental characters and the results are not consistent across datasets. Molecular benchmarks (being entirely independent of 
46 the character data) provide a framework for examining each dataset individually to assess the

47 evolution of the characters used.

48

49 Keywords: Evolutionary Rates; Homoplasy; Independence; Phylogeny; Mammalia

50

51

52

53

54

INTRODUCTION

Accurate reconstructions of phylogenetic relationships are essential for studying the evolutionary history of a clade, with hypotheses being based on molecular or morphological data, or both. While it is comparatively straightforward to observe patterns of evolution in molecular sequence data and therefore develop models more closely representing the evolutionary processes, this is more difficult in the case of morphological characteristics due to a poorer understanding of how novel morphology is evolved from ancestral traits. Nonetheless, morphological data are our only means of reconstructing the phylogenetic relationships of fossil organisms that are too old to preserve DNA or usable proteins. It is therefore imperative that we strive to better understand the evolutionary modes of morphological traits. In recent years many studies have examined how variation in evolutionary patterns of discrete morphological traits, not accounted for by current analyses, may be affecting phylogenetic inferences (e.g. O'Keefe \& Wagner, 2001; Scotland et al., 2003; DeGusta, 2004; Sansom et al. 2017; Billet \& Bardin, 2018). The high percentage of dental characters used in the reconstruction of fossil mammal phylogenies has become a particular cause for concern. Numerous studies have highlighted issues such as the non-independent evolution of dental characters (Kangas et al., 2004; Kavanagh et al., 2007; Harjunmaa et al., 2014; Dávalos et al., 2014; Billet \& Bardin, 2018) and increased convergence relative to other character partitions due to ecological selective pressures (Evans et al., 2007; Kavanagh et al., 2007). In a recent meta-analysis, Sansom et al. (2017) examined the 
69 phylogenetic signal of tooth and osteological character partitions, using phylogenies derived

70 from molecular data as a benchmark. This study found that osteological characters were more

71 consistent with the molecularly-derived phylogenies and contained greater phylogenetic signal

72 than dental characters. Further, parsimony analyses with only dental characters produced results

73 less similar to the molecularly-derived phylogenies than analyses where the same number of

74 characters were selected at random from both partitions (Sansom et al., 2017).

75 This paper builds on the work of Sansom et al. (2017) in two principal ways. Sansom et

al. (2017) employed two partitions, dental and osteological, to assess the performance of dental

77 characters relative to osteological characters in phylogenetic analyses. As such, while dental

characters have been demonstrated to be problematic, an understanding of whether this problem

was limited to them, or whether it extends to other partitions, is lacking. We therefore examine

phylogenetic signal in six morphological partitions in mammals in order to establish whether any

81 other skeletal regions may be a poor indicator of phylogeny.

Secondly, we also aim to understand why dental characters may be producing

phylogenies less congruent with molecularly-derived benchmarks. Many studies have established

that morphological characters frequently violate at least some of the principal assumptions of

parsimony (see below): between-character rate homogeneity (all characters being just as likely to

transition), within-character rate homogeneity (all character states within the same character

being similarly likely to transition), and character independence. We test each morphological character partition for variation in these parameters.

In most published phylogenetic analyses performed using parsimony, the characters are

91 scheme, a change in any character is given equal influence in determining tree length. However, 
92 such a scheme only produces robustly supported results when the characters are all equally likely

93 to change. If, however, there is variation in the rates of character evolution, certain characters

94 will change more frequently and are more likely to show homoplasy (Felsenstein, 1981;

95 Goloboff, 1993). While parsimony analysis does not incorporate an explicit evolutionary model,

96 an equal weights analysis does rely on equal between-character rates for its accuracy.

97 Furthermore, in most published phylogenetic analyses, transitions between different

98 combinations of character states are given equal weight (i.e. a transition from state 0 to state 1 is

99 just as likely as a transition from state 1 to state 0 ; an assumption of within-character rate

100 homogeneity). This assumption may be relaxed by incorporating step matrices which give

101 greater weight to particular transitions (Sankoff \& Cedergren, 1983), or by ordering (Fitch,

102 1971), an extreme modification of step matrices, setting the possibility of non-adjacent

103 transitions to 0 . However, step matrices are rarely employed, and the use of ordered characters is

104 still heavily debated (see Marjanović \& Laurin, 2019 for summary of their history), so most

105 analyses assume equality of within-character rates.

106 Finally, all methods of phylogenetic analysis (parsimony, Bayesian, and likelihood), treat

107 all characters as independent of one another (i.e. an assumption that a change in one character

108 will have no effect on the transition probability in another character). Extensive study has shown

109 this assumption of independence to be frequently violated (e.g. Kangas et al., 2004; Kavanagh et

110 al., 2007; Harjunmaa et al., 2014; Dávalos et al., 2014; Billet and Bardin, 2018), with many traits

111 or regions forming integrated modules that change as a unit (Goswami, 2006, 2007; Goswami \&

112 Polly, 2010).

113 By analysing phylogenetic signal, between- and within-character rates, and character

114 independence across six morphological partitions, within mammals as a whole and within four 
115 mammalian subclades, we aim to better understand how morphological characters can be

116 selected and formulated during phylogenetic analyses of mammals. The results should provide

117 future studies that intend to reconstruct the relationships of fossil mammals with a framework to

118 enable more evidence-based decisions about which characters are more reliable for use in

119 phylogenetic analyses.

120

121 MATERIALS AND METHODS

122 Data

123 This study builds on the protocol established by Sansom et al. (2017), where molecularly-

124 derived phylogenies are used as the framework over which morphological evolution may be

125 analysed. This allows the evolutionary patterns of the characters to be examined over a

126 phylogeny produced and time calibrated from data entirely independent of those characters.

127 Unlike Sansom et al. (2017), all phylogenies used were also scaled to time using molecular data,

128 as required by the phylogenetic comparative methods employed. For mammals the time-scaled

129 molecularly-derived phylogeny was taken from Dos Reis et al. (2012), and the morphological

130 data from Bi et al. (2014), both recent and comprehensive datasets. Although the Bi et al. (2014)

131 matrix was focussed on Mesozoic mammals, it contains a broad sampling of modern clades,

132 including taxa from the monotremes, marsupials and placentals. The morphological characters

133 were divided between six partitions: dental, cranial, axial, forelimb (including pectoral girdle),

134 hindlimb (including pelvic gridle), and soft tissue. Taxa not present in both the morphological

135 matrix and molecularly-derived tree were dropped. If, after doing so, a character showed no

136 variation in score among the remaining taxa, that character was also dropped from subsequent

137 analyses. 
As well as the global analysis of mammals, four subclades were subjected to the same

139 analyses to test for variation in the macroevolutionary patterns within Mammalia. The clades

140 chosen were as follows: Artiodactyla (Molecularly-derived tree from Hassanin et al. [2012],

141 Morphological matrix from Spaulding et al. [2009]), Carnivora (Molecularly-derived tree from

142 Eizirik et al. [2010], Morphological matrix from Tomiya [2011]), Primates (Molecularly-derived

143 tree from Perelman et al. [2011], Morphological matrix from Ni et al. [2013]) and Marsupialia

144 (Molecularly-derived tree from Mitchell et al. [2014], Morphological matrix from Beck [2017]).

145 These clades were chosen for the following reasons: 1) they have been analysed using

146 morphological character matrices containing characters from all six of the morphological

147 partitions; 2) there exist time calibrated molecularly-derived phylogenies with substantial

148 taxonomic overlap with the morphological matrices; 3) the character list, data matrix and time

149 calibrated phylogeny were available in usable formats; and 4) they are morphologically and

150 ecologically diverse lineages, and therefore the morphological characters have the potential to be

151 heavily influenced by functional and ecological constraints.

153 Phylogenetic Signal

154 Levels of homoplasy relative to the molecularly-derived phylogeny were used as an

155 estimate of the phylogenetic signal of the characters, measured using Pagel's lambda (Pagel,

156 1999), a metric shown to perform well under simulations (Münkemüller et al., 2012). This

157 statistic produces a value between 0 and 1 , where 0 indicates that character states are distributed

158 independent of phylogeny (no phylogenetic signal). Other methods of calculating phylogenetic

159 signal in discrete characters, for example Moran's I (Gittleman \& Kot, 1990) or Fritz \& Purvis’s

160 D (Fritz \& Purvis, 2010), were not used as they are only suitable for binary characters and would 
161 require a large proportion of the characters to be dropped. For each character, taxa scored as

162 unknown were dropped from the tree. If more than a quarter of the taxa were scored as unknown,

163 the character was not considered in this or subsequent analyses. Pagel's lambda was calculated in

$164 \mathrm{R}$ version 3.3.2 (R Core Team, 2016) using the fitDiscrete function in the package geiger

165 (Harmon et al., 2007).

166

167 Testing the Assumptions of Phylogenetic Analysis

168 Within-character rate homogeneity was tested by fitting models of discrete character

169 evolution to the observed phylogeny and trait values using the function fitDiscrete in the $\mathrm{R}$

170 package geiger. This method calculates the likelihood of a particular model based on the data,

171 and also estimates the values of variable parameters within that model that best fits the observed

172 data (Pennell and Harmon 2013, Pennell et al. 2014). Two models were compared: an equal rates

173 (ER) model, where every possible character state transformation has the same rate, and an all-

174 rates-different (ARD) model, where every possible character state transformation is allowed a

175 different rate. The models are compared using the Akaike information criterion, which penalises

176 the parameter-rich ARD model. The Akaike weights of the ER model are used as a metric to

177 assess how well a character obeys the assumption of within-character rate homogeneity.

178 The fitDiscrete function also allows testing of between-character rate homogeneity. As

179 mentioned above, as well as identifying the model of discrete character evolution that best fits

180 the trait and phylogeny, it also identifies the rates of character-state transformation that best fits

181 the observed data. A higher rate of change means a character is more likely to change multiple

182 times by convergence. If a character was found to best fit the ER model in the above analysis,

183 then the single rate of change was assigned to the character. If the ARD model was found to fit 
184 best, the rate assigned to that character was the mean of all rates assigned to each possible

185 transformation, weighted by the number of times each transformation occurred over the

186 phylogeny. The number of transitions was inferred by stochastically mapping the character over

187 the phylogeny 1000 times using the make.simmap function in in the R package phytools (Revell, 188 2012), and calculating the mean frequency of each possible transition.

189 To test character independence, the method of Pagel (1994) was applied to pairwise

190 comparisons of characters. This is again a model-fitting approach, where non-independent and

191 independent models of character evolution are fit to pairs of traits and the observed phylogeny.

192 Under the non-independent model, the rate of character change in trait 1 will depend on which

193 character state is observed in trait 2, and vice versa. Under the independent model, both

194 characters change state independently of each other. Again, the two models may be compared via

195 the Akaike information criterion, and the Akaike weights of the independent model may be used

196 as a metric for how well a pair of characters obeys the assumption of independent evolution.

197 Unfortunately, this method is only applicable to binary characters, so non-binary characters were

198 not considered in this section of the analyses. The analysis was implemented using the function

199 fitPagel in phytools.

200

201 Statistical Comparisons

202 Pagel's lambda values for each character partition were compared using generalised least 203 squares (GLS), using the R package nlme (Pinheiro et al 2017). For each partition, a null model 204 where all the phylogenetic signal of all partitions comes from the same distribution, was 205 compared to a model where only the partition of interest had a different phylogenetic signal 206 (H1). The Akaike weights was used to infer which best fit the data. Partitions that better fit the 
207 H1 model were deemed to have significantly different phylogenetic signals than the other

208 partitions, with the GLS coefficient used to identify whether (positive coefficient) or lower

209 (negative coefficient). The same method was also applied to the rate values, the support for the

210 ER model, and support for the independent model of evolution.

211 The rate of character change for each character, and the Akaike weight for the ER model

212 for each character, were both compared to Pagel's lambda using the Kendall's tau correlation

213 coefficient, a non-parametric method that does not assume normality of the data. This latter test

214 could not be applied to the Akaike weights values of the independent model of evolution because

215 these represent pairwise comparisons of characters rather than individual characters. The number

216 of characters in partitions was compared to the median phylogenetic signal, median rate, median

217 support for the ER model and median support for the independent model to test the influence of

218 character sampling on the results.

220

RESULTS

221

Results from the Total Mammalia Dataset

222

The median phylogenetic signal calculated from the Bi et al. (2014) character matrix (the

223

total Mammalia dataset) was 1 for all partitions (white points, Fig. 1A). This indicates that at

224 least half of the characters in each partition are synapomorphies for a single clade. The dental

225 characters do show a larger range and interquartile range of lambda values than the other

226 partitions. However, the range of values observed for cranial characters is similar to that of

227 dental characters. In the GLS analysis, cranial characters are the only partition to not better fit the

228 null model; instead they are found to have significantly lower phylogenetic signal than other

229 partitions (Table 1). 
231 do the other partitions (Fig. 1B). In fact, the Akaike weights of the equal rates (ER) model are

232 the highest of all the partitions, and in the GLS analysis no partitions have significantly better

233 support for the ER model than other partitions (Table 2). Dental characters also show no

234 evidence of increased non-independence relative to other partitions (Fig. 1C). Only the forelimb

235 partition was found to have significantly worse support for the independent model of evolution

236 than other partitions (Table 3). The hindlimb was found to have significantly better support for

237 the independent model.

238 However, dental characters have the highest median rates of evolution compared to all

239 other partitions (Fig. 1D), and the increase in rates is significant according to the GLS analysis

240 (Table 4). No other partitions were found to have a significant difference in rate relative to the 241 null model.

Results from Mammalian Subclade Datasets

The Artiodactyla datasets produced similar results to those of mammals overall, albeit with considerably more variation in phylogenetic signal from the vertebral, forelimb and soft tissue characters (Fig. 2A). The dental characters are the only partition where the GLS analysis found phylogenetic signal to be significantly reduced relative to other partitions (Table S1). Rates of dental evolution are again significantly higher than for other partitions (Fig. 2D, Table S4). There is no significant difference found between the Akaike weights support for the ER model of evolution in teeth (Table S2), nor the support for the independent model of character

251 evolution, compared to other partitions (Table S3). The skull partition shows better support for 
252 the independent model, while the forelimb shows statistically significantly reduced

253 independence.

254 The carnivoran dataset also found dental characters to have significantly lower

255 phylogenetic signal than other partitions (Fig 3A, Table S7). In this clade the dental character

256 partition also has higher rates than all other partitions except the forelimb (for which there is only 257 one character) (Fig 3D).

258 The primate and marsupial datasets produced results conflicting with the other two 259 subclades and mammals as a whole (Figs 4,5). The dental partition in primates has significantly

260 higher phylogenetic signal and significantly lower rates of evolution than other partitions (Fig 4,

261 Tables S9, S12). The dental partition also had significantly better support for the equal rates

262 model of evolution than other partitions. However, primate characters suffer from being highly

263 integrated: all partitions other than the vertebrae and soft tissue characters show a significantly

264 low fit to the independent model of evolution (Fig 4C, Table S11).

265 In marsupials, while many of the character partitions, including dentition, show a wide

266 range of Pagel's lambda values, the lambda values of the tooth characters are more concentrated

267 towards higher values compared other partitions (Fig 5A). The tooth characters show no

268 significant difference in their phylogenetic signal relative to other partitions (Table S13). The

269 dental characters showed no significant difference from any other partitions in support for the ER

270 model of evolution (Table S14), and no significant difference in rates (Fig. 5D). In contrast to the

271 other datasets, however, the marsupial dataset does support increased character non-

272 independence of dental characters relative to other partitions, with median Akaike weights

273 support for the independent model of evolution lower than all other partitions (Fig. 5C; Table 274 S15). 
276

277

278

279

280 281 (Table 6).

282

283

284

285

286

287

288

289

290

291

292

293

294 295

\section{DISCUSSION}

Correlation Tests

In all five datasets, there is a negative correlation between lambda and rate of character evolution, significant in all except Carnivora (Table 5). The correlation between the lambda values and Akaike weights of the ER model is weaker in all five, but in some is still significant. None of the parameters tested correlated significantly with number of characters in each partition

Mammalian tooth characters have been a source of much discussion over the last two decades, due in part to their dominance of the character lists used in morphological phylogenetic analyses of mammals, itself to an extent a product of their dominance in the mammalian fossil record. Teeth have been shown to suffer from issues such as large amounts of homoplasy (Evans et al., 2007; Davalos et al., 2014) and non-independence (Kangas et al., 2004; Harjunmaa et al., 2014). While these issues clearly do impact on the utility of dental characters in phylogenetic analysis, what has received less attention is whether dental characters are in fact worse affected than other body partitions in these regards. The majority of studies cited above focus solely on teeth, but issues of homoplasy due to ecological and functional constraints might be expected to affect other character partitions (e.g. limb characters being functionally linked to locomotion). Indeed, ecological constraint and developmental linkage has been demonstrated in cranial and limb characters across various tetrapod groups, including mammals (Ruvinsky \& Gibson-Brown, 2000; Young \& Hallgrímson, 2005; Sadleir \& Mackovicky, 2008). The same argument could be made for the issue of character non-independence: while this has been demonstrated to be a 
298 problem with mammal dentition, recent work on modularity and integration highlights that this

299 issue might just as strongly impact on non-dental characters (Goswami 2006, 2007; Goswami \&

300 Polly 2010).

301 Our analyses suggest that increased homoplasy driven by increased rates of evolution

302 may affect dental characters to a greater extent than other partitions. Dental characters from the

303 total Mammalia dataset and the artiodactyl and carnivoran datasets are found to evolve at faster

304 rates than the other character partitions, and so are more likely to transition multiple times. The

305 strong and significant inverse correlations between phylogenetic signal and rates of evolution in

306 all tested datasets indicates that rate variation is likely to be the main driving force behind loss of

307 phylogenetic signal, more so than within-character rate heterogeneity. However, this signal is not

308 consistent across all the tested clades. In the marsupial and primate datasets, dental characters

309 have lower rates (and higher phylogenetic signal) than most other partitions.

310 Moreover, while the results obtained here seem to suggest that dental characters have

311 lower phylogenetic signal than some other characters when optimised over a molecular-based

312 phylogeny, they are not alone in this respect. The total Mammalia dataset indicates that cranial

313 characters also have low phylogenetic signal. In both primates and marsupials, the soft tissue

314 characters have lower Pagel's lambda values than any other partition (Fig 4A, 5A) and in

315 carnivorans both limb partitions perform poorly in this respect (Fig 3A).

316 The results observed in artiodactyls raise a possibility that might warrant future study: the

317 increase in rates of dental evolution observed might be due to the dominance of herbivores in this

318 dataset. Herbivory has been suggested to be a driver of dental disparity in mammals (Jernvall et

319 al., 1996, 2000) as their morphology tracks a constantly changing resource (plants). Since the

320 functional requirements of eating meat has not changed over time, carnivorous mammals show 
321 reduced dental disparity and less evolutionary change (Van Valkenburgh, 1988; Wesley-Hunt et

322 al., 2005). In an analysis of diversification patterns across all mammals, herbivores showed

323 significantly higher diversification rates than carnivores or omnivores (Price et al. 2012). While

324 this analysis focussed on lineage diversification, the authors cited increased specialisation and

325 niche-subdivision as a potential driving force behind diversification patterns, and morphological

326 diversification patterns should respond to these drivers in the same way.

327 It is finally worth noting that in the total-Mammalia dataset and two of the three placental

328 subclades tested, there is little evidence that tooth characters are affected by non-independence to

329 any greater extent than the other morphological partitions. The primate and marsupial datasets

330 are the exception, with dental characters showing a weaker fit to the independent model than all

331 other partitions. The integration of the dental characters and their low rates of evolution in

332 marsupials may be due to the unusual development: neonatal marsupials, born extremely early in

333 their development need to attach to the teat, leading to precocial development of the jaw and

334 facial region in marsupials (Smith, 1996, 2006). This could lead to this region evolving as a more

335 integrated module. Alternatively, it may be a result of character selection: the Beck (2017)

336 dataset contains large numbers of characters relating to the presence or absence or particular

337 dental loci in both upper and lower jaws, likely to be heavily integrated.

339 Conclusions

340 The concept pioneered by Sansom et al. (2017), of testing morphological discrete

341 characters over a molecular benchmark, is a powerful tool, and it would be highly recommended

342 that researchers studying clades where molecularly-derived phylogenies exist examine the

343 performance of their characters in this manner. But given the extremely wide variation in results 
344 found by this study, where different partitions produced different relative phylogenetic signals

345 (with the primates and marsupials in particular producing results conflicting strongly with the

346 other datasets studied), one should perhaps be cautious of basing assumptions of character

347 quality on the results of large meta-analyses. While the latter are useful for identifying broad-

348 scale patterns, it is necessary that each dataset be examined individually, and decisions made

349 based on the macroevolutionary patterns observed in that clade. However, larger-scale meta-

350 analyses do have the advantage in that they are less likely to be affected by idiosyncrasies of

351 individual datasets and the choices/intentions of the researchers. For example, the total-

352 Mammalia dataset includes only three soft-tissue characters relating to the different modes of

353 reproduction, likely intended to separate monotremes, marsupials and placentals, and therefore

354 this likely is not representative of the total variation in soft tissue evolution. A detailed

355 examination of specific characters and how variation in their formulation affects the results

356 would be an avenue for future research

357 A fair and comprehensive sampling of characters across partitions should be the aim;

358 experiments incorporating random sampling of characters show that sampling across partitions

359 leads to a more reliable estimation of phylogenetic relationships than sampling within single

360 partitions (Pattinson et al., 2015). While dental characters have been shown to suffer from issues

361 of homology and non-independence (Kangas et al., 2004; Evans et al., 2007; Harjunmaa et al.,

362 2014), the comparison of the dental characters to finer partitions of data presented here

363 demonstrates that these issues are not unique to teeth. In fact, in some cases other regions

364 perform even worse, and the nature of these issues varies from clade to clade.

365

366 ACKNOWLEDGMENTS

Peer] reviewing PDF | (2019:09:41216:2:0:NEW 29 Jan 2020) 
We would like to thank Roger Benson and Robert Sansom for helpful discussion. Robert 368 Asher, Robin Beck and an anonymous reviewer provided helpful comments on an early draft of

369 the manuscript. NB's research is funded by Deutsche Forschungsgemeinschaft grant number BR

$3705724 / 1-1$. GLB's research is funded by a NERC studentship from the Oxford DTP in

371 Environmental Research (NE/L0021612/1), alongside additional support provided by the

372 European Research Council (grant agreement 637483).

373

374

\section{REFERENCES}

375 Beck R. M. 2017. The skull of Eepidolops ameghinoi from the early Eocene Itaboraí fauna,

376 southeastern Brazil, and the affinities of the extinct marsupialiform order

377 Polydolopimorphia. Journal of Mammalian Evolution, 24, 373-414

Bi S., Wang Y., Guan J., \& Meng J. 2014.Three new Jurassic euharamiyidan species reinforce early divergence of mammals. Nature, 514, 579-584.

Billet G. \& Bardin J. 2018. Serial homology and correlated characters in morphological phylogenetics: modelling the evolution of dental crests in placentals. Systematic Biology, In Press

Dávalos L. M., Velazco P. M., Warsi O. M., Smits P. D., \& Simmons N. B. 2014. Integrating incomplete fossils by isolating conflicting signal in saturated and non-independent morphological characters. Systematic Biology, 63, 582-600.

DeGusta D. 2004. A method for estimating the relative importance of characters in cladistic 387 analyses. Systematic Biology, 53, 529-532 
388 Dos Res M., Inoue J., Hasegawa M., Asher R. J., Donoghue P. C. J., \& Yang Z. 2012.

389

390

391 Eizirik E., Murphy W. J., Koepfli K.-P., Johnson W. E., Dragoo J. W., Wayne R. K., \& O’Brien
Phylogenomic datasets provide both precision and accuracy in estimating the timescale of placental mammal phylogeny. Proceedings of the Royal Society B, 279, 3491-3500

395

396

397

398

399

400

401

402

403

404

405

406 inferred from multiple nuclear gene sequences. Molecular Phylogenetics and Evolution, $56,49-63$

Evans A. R., Wilson G. P., Fortelius M., \& Jernvall J. 2007. High-level similarity of dentitions in carnivorans and rodents. Nature, 445, 78-81.

Felsenstein J. 1981. Evolutionary trees from DNA sequences: a maximum likelihood approach. Journal of Molecular Evolution, 17, 368-376

Fitch W. M. 1971. Toward defining the course of evolution: minimum change for a specific tree topology. Systematic Biology, 20, 406-416

Fritz S. A., \& Purvis A. 2010. Selectivity in mammalian extinction risk and threat types: a new measure of phylogenetic signal strength in binary traits. Conservation Biology, 24, 10421051.

Gittleman J. L. \& Jot M. 1990. Adaptation: statistics and a null model for estimating phylogenetic effects. Systematic Zoology, 39, 227-241

Goloboff, P.A. 1993. Estimating character weights during tree search. Cladistics, 9, 83-91 
407 Goloboff P.A, Carpenter J.M., Arias J.S. \& Esquivel, D.R.M. 2008. Weighting against

408 homoplasy improves phylogenetic analysis of morphological datasets. Cladistics, 24, $409 \quad 758-773$.

410 Goswami A. 2006. Cranial modularity shifts during mammalian evolution. American Naturalist, $168,270-280$.

412 Goswami A. 2007. Cranial modularity and sequence heterochrony in mammals. Evolution and 413 Development, 9, 290-298.

414 Goswami A. \& Polly P. D. 2010. The influence of modularity on cranial morphological disparity in Carnivora and Primates (Mammalia). PLoS ONE, 5, e9517.

Harjunmaa E., Seidel K., Häkinen T., Renvoisé, Corfe I.J., Kallonen A., Zhang Z.Q., Evans A.R., Mikkola M.L., Klein O.D., \& Jernvall J. 2014. Replaying evolutionary transitions from the dental fossil record. Nature, 512, 44-48.

Harmon L. J., Weir J. T., Brock C. D., Glor R. E., \& Challenger W. 2007. GEIGER: investigating evolutionary radiations. Bioinformatics, 24, 129-131

Hassanin A., Delsuc F., Ropiquet A., Hammer C., van Vuuren B. J., Matthee C., Ruiz-Garcia M., Catzeflis F., Areskoug V., Nguyen T. T., \& Couloux A. 2012. Pattern and timing of diversification of Cetartiodactyla (Mammalia, Laurasiatheria), as revealed by a comprehensive analysis of mitochondrial genomes. Comptes Rendus Biologie, 335, 3250.

Jernvall J., Hunter J. P., \& Fortelius M. 1996. Molar tooth diversity, disparity, and ecology in Cenozoic ungulate radiations. Science, 274, 1489-1492. 
428 Jernvall J., Hunter J. P., \& Fortelius M. 2000. Trends in the evolution of molar crown types in 429 ungulate mammals: evidence from the Northern Hemisphere. In Teaford M. F., Smith M.

430 M., \& Ferguson M. W. J. (eds) Development, Function and Evolution of Teeth (p.p. 269431 281). Cambridge University Press, Cambridge.

432 Kälersjö M., Albert, V. A., \& Farris J. S. 1999. Homoplasy increases phylogenetic structure. $433 \quad$ Cladistics, $15,91-93$

434 Kavanagh K. D., Evans A. R. \& Jernvall J., 2007. Predicting evolutionary patterns of 435 mammalian teeth from development. Nature, 449, 427-432.

436 437 438
Kangas A. T., Evans A. R., Thesleff I., \& Jernvall J. 2004. Nonindependence of mammalian dental characters. Nature, 432, 211-214.

Kluge A. G. 2005. What is the rationale for 'Ockham's razor' (aka parsimony) in phylogenetic inference? In Albert, V. A. (ed) Parsimony, Phylogeny and Genomics (pp. 15-42). Oxford University Press, Oxford

Marjanović, D., \& Laurin M. 2019. Phylogeny of Palaeozoic limbed vertebrates reassessed through revision and expansion of the largest published relevant data matrix. PeerJ, 6, e5565

Mitchell K. J., Pratt R. C., Watson L. N., Gibb G. C., Llamas B., Kasper M., Edson J., Hopwood B., Male D., Armstrong K. N., Meyer M., Hofreiter M., Austin J., Donnellan S. C., Lee M. S. Y., Philips M. J., \& Cooper A. 2014. Molecular phylogeny, biogeography, and habitat preference evolution of marsupials. Molecular Biology and Evolution, 31, 23222330. 
449 Münkemüller T., Lavergne S., Bzeznik B., Dray S., Jombart T., Schiffers K., Thuiller W. 2012. $450 \quad$ How to measure and test phylogenetic signal. Methods in Ecology and Evolution, 3, 743451 56.

452 Ni X., Gebo D. L., Dagosto M., Meng J., Tafforeau P., Flynn J. J., Beard K. C. 2013 The oldest 453 known primate skeleton and early haplorhine evolution. Nature, 498, 60.

454 O’Keefe F. R., \& Wagner P. K. 2001. Inferring and testing hypotheses of cladistic character 455 dependence by using character compatibility. Systematic Biology, 50, 657-675.

456

458

460

461

462

463

464

465

466

467

468

Pagel M. 1999. Inferring the historical patterns of biological evolution. Nature, 401, 877-884.

Pattinson D. J., Thompson R. S., Piotrowski A. K., \& Asher R. J. 2015. Phylogeny, paleontology, and primates: do incomplete fossils bias the Tree of Life? Systematic Biology, 64,169-186

Pennell M. W., \& Harmon M. J. 2013. An integrative view of phylogenetic comparative methods: connections to population genetics, community ecology and paleobiology. Annals of the New York Academy of Sciences, 1289, 90-105

Pennell M. W., Eastman J. M., Slater G. J., Brown J. W., Uyeda J. C., FitzJohn R. G., Alfaro M. E., \& Harmon L. J. 2014. Geiger v2.0: an expanded suite of methods for fitting macroevolutionary models to phylogenetic trees. Bioinformatics, 30, 2216-2218. 
469 Perelman P., Johnson W. E., Roos, C., Seuánez H. N., Horvath J. E., Moreira M. A. M., Kessing B., Pontius J., Roelke M., Rumpler Y., Schneider M. P. C., Siva A., O’Brien S. J., \& Pecon-Slattery J. 2011. A molecular phylogeny of living primates. PLoS Genetics, 7,

Pinheiro J., Bates D., DebRoy S., Sarkar D., Heisterkamp S., \& Van Willigen B. 2017. Package $\mathrm{e} 1001342$

Price S. A., Hopkins S. S. B., Smith K. K. \& Roth, V. L. 2012. Tempo of trophic evolution and its impact on mammalian diversification. Proceedings of the National Academy of Science, 109, 7008-7012.

R Core Team. 2016. R: a language and environment for statistical computing. Vienna, Austria.

479

480

481 482

$$
\text { 'nlme'. Linear and Nonlinear Mixed Effects Models, version 3.1 }
$$

Revell L. J. 2012. phytools: an R package for phylogenetic comparative biology (and other things. Methods in Ecology and Evolution, 3, 217-223

Ruvinksy I., \& Gibson-Brown J. J. 2000. Genetic and developmental bases of serial homology in vertebrate limb evolution. Development, 127, 5233-5244.

Sadleir R. W., \& Makovicky P. J. 2008. Cranial shape and correlated characters in crocodilian evolution. Journal of Evolutionary Biology, 21, 1578-1596.

Sankoff D., \& Cedergren R. J. 1983. Simultaneous comparison of three or more sequences related by a tree. In Sankoff, D., Kruskal, J.B. (eds). Time Warps, String Edits, and Macromolecules: The Theory and Practice of Sequence Comparison (pp. 253-263). Addison-Wesley, Reading, Massachusetts 
489 Sansom R. S., Wills M. A., \& Williams T. 2017. Dental data perform relatively poorly in 490 reconstructing mammal phylogenies: morphological partitions evaluated with molecular 491 benchmarks. Systematic Biology, 66, 813-822.

492 Scotland R. W., Olmstead R. G., \& Bennett J. R. 2003. Phylogeny reconstruction, the role of 493 morphology. Systematic Biology, 52, 529-548.

494 Smith K. K. 1996. Integration of craniofacial structures during development in mammals. American Zoologist, 26, 70-79

496

497

498

499

500

501

502

503

504

505

506

507 508 509

Smith K. K. 2006. Craiofacial development in marsupial mammals: developmental origins of evolutionary change. Developmental dynamics, 235, 1181-1193

Spaulding M., O'Leary M. A, \& Gatesy J. 2009. Relationships of Cetacea (Artiodactyla) among mammals: increased taxon sampling alters interpretations of key fossils and character evolution. PLoS ONE, 4, e7062.

Tomiya S. 2010. A new basal caniform (Mammalia: Carnivora) from the middle Eocene of North America and remarks on the phylogeny of early carnivorans. PLoS ONE, 6, e24146

Van Valkenburgh B. 1988. Trophic diversity in past and present guilds of large predatory mammals. Paleobiology, 14, 155-173

Wesley-Hunt G. D. 2005. The morphological diversification of carnivores in North America. Paleobiology, 31, 35-55

Young N., \& Hallgrímsson B. 2005. Serial homology and the evolution of mammalian limb covariation structure. Evolution, 59, 2691-2704. 


\section{FIGURE CAPTIONS}

512 Figure 1: Violin plots illustrating results from the Bi et al. (2014) character matrix (total

513 Mammalia). A) Pagel's lambda values (phylogenetic signal) of each character. A value of 0

514 indicates no phylogenetic signal, while a value of 1 indicates high phylogenetic signal. B)

515 Akaike weights support for the ER model of evolution of each character. Characters with an

516 Akaike weights score of 1 have equal rates of within-character evolution between each state,

517 while characters with a score of 0 display unequal rates of within-character state evolution. C)

518 Akaike weights support for the independent model of evolution of all pairwise comparisons of

519 characters in each partition. Pairwise comparisons that have an Akaike weights score of 1 evolve

520 independently of one another, while pairwise comparisons with a score of 0 display character

521 non-independence. D) Rates of character evolution of each character (log10 transformed). The

522 number of characters in each partition can be found at the base of the figure $(n=\mathrm{X})$. For each

523 partition, the horizontal spread of the violin plot represents the density of data at each point on

524 the y-axis. Box plots with a white point representing the median are plotted within each violin

525 plot. The heatmap is a visual representation of the y-axis.

526

527 Figure 2: Violin plots illustrating results from Spaulding et al. (2009) matrix (Artiodactyla). A)

528 Pagel's lambda values (phylogenetic signal) of each character. B) Akaike weights support for the

529 ER model of evolution of each character. C) Akaike weights support for the independent model

530 of evolution of all pairwise comparisons of characters in each partition. D) Rates of character

531 evolution of each character ( $\log 10$ transformed). The number of characters in each partition can

532 be found at the base of the figure $(n=\mathrm{X})$ 
534 Figure 3: Results from the Tomiya (2011) matrix (Carnivora). A) Pagel's lambda values

535 (phylogenetic signal) of each character. B) Akaike weights support for the ER model of

536 evolution of each character. C) Akaike weights support for the independent model of evolution

537 of all pairwise comparisons of characters in each partition. D) Rates of character evolution of

538 each character $(\log 10$ transformed). The number of characters in each partition can be found at

539 the base of the figure $(n=\mathrm{X})$

540

541 Figure 4: Violin plots illustrating results from the Ni et al. (2013) matrix (Primates). A) Pagel's

542 lambda values (phylogenetic signal) of each character. B) Akaike weights support for the ER

543 model of evolution of each character. C) Akaike weights support for the independent model of

544 evolution of all pairwise comparisons of characters in each partition. D) Rates of character

545 evolution of each character ( $\log 10$ transformed). The number of characters in each partition can

546 be found at the base of the figure $(n=\mathrm{X})$

547

548 Figure 5: Violin plots illustrating results from the Beck (2017) matrix (Marsupialia). A) Pagel's

549 lambda values (phylogenetic signal) of each character. B) Akaike weights support for the ER

550 model of evolution of each character. C) Akaike weights support for the independent model of

551 evolution of all pairwise comparisons of characters in each partition. D) Rates of character

552 evolution of each character ( $\log 10$ transformed). The number of characters in each partition can

553 be found at the base of the figure $(n=\mathrm{X})$ 


\section{Figure 1}

Figure 1: Violin plots illustrating results from the Bi et al. (2014) character matrix (total Mammalia)

A) Pagel's lambda values (phylogenetic signal) of each character. A value of 0 indicates no phylogenetic signal, while a value of 1 indicates high phylogenetic signal. B) Akaike weights support for the ER model of evolution of each character. Characters with an Akaike weights score of 1 have equal rates of within-character evolution between each state, while characters with a score of 0 display unequal rates of within-character state evolution. C) Akaike weights support for the independent model of evolution of all pairwise comparisons of characters in each partition. Pairwise comparisons that have an Akaike weights score of 1 evolve independently of one another, while pairwise comparisons with a score of 0 display character non-independence. D) Rates of character evolution of each character ( $\log 10$ transformed). The number of characters in each partition can be found at the base of the figure $(n=\mathrm{X})$. For each partition, the horizontal spread of the violin plot represents the density of data at each point on the $y$-axis. Box plots with a white point representing the median are plotted within each violin plot. The heatmap is a visual representation of the $y$ axis. 

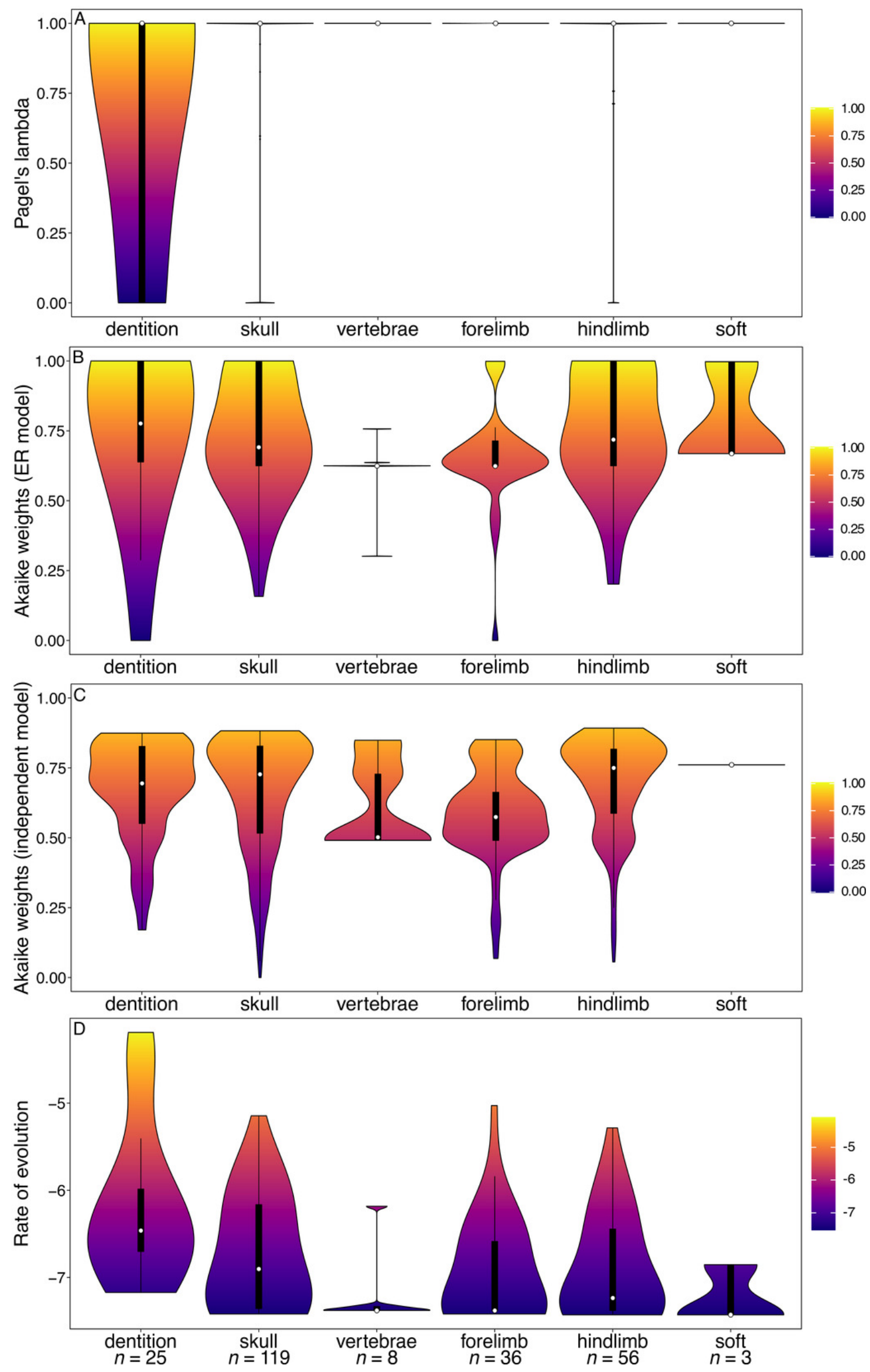

Peer] reviewing PDF | (2019:09:41216:2:0:NEW 29 Jan 2020) 


\section{Figure 2}

Figure 2: Violin plots illustrating results from Spaulding et al. (2009) matrix (Artiodactyla).
A) Pagel's lambda values (phylogenetic signal) of each character. B) Akaike weights support for the ER model of evolution of each character. C) Akaike weights support for the independent model of evolution of all pairwise comparisons of characters in each partition. D) Rates of character evolution of each character (log10 transformed). The number of characters in each partition can be found at the base of the figure $(n=X)$ 

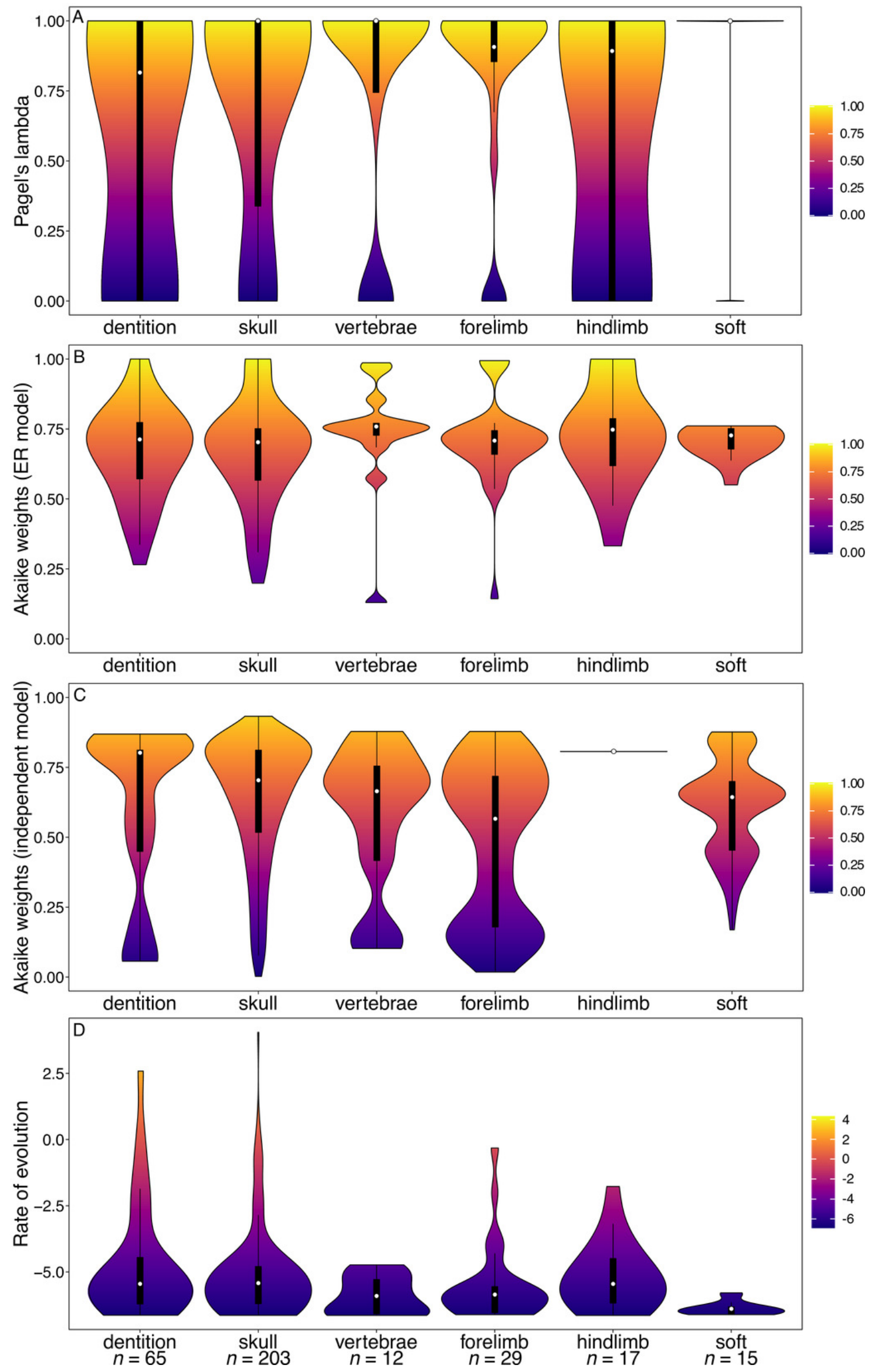

Peer) reviewing PDF | (2019:09:41216:2:0:NEW 29 Jan 2020) 


\section{Figure 3}

Figure 3: Results from the Tomiya (2011) matrix (Carnivora).

A) Pagel's lambda values (phylogenetic signal) of each character. B) Akaike weights support for the ER model of evolution of each character. C) Akaike weights support for the independent model of evolution of all pairwise comparisons of characters in each partition. D) Rates of character evolution of each character (log10 transformed). The number of characters in each partition can be found at the base of the figure $(n=\mathrm{X})$ 

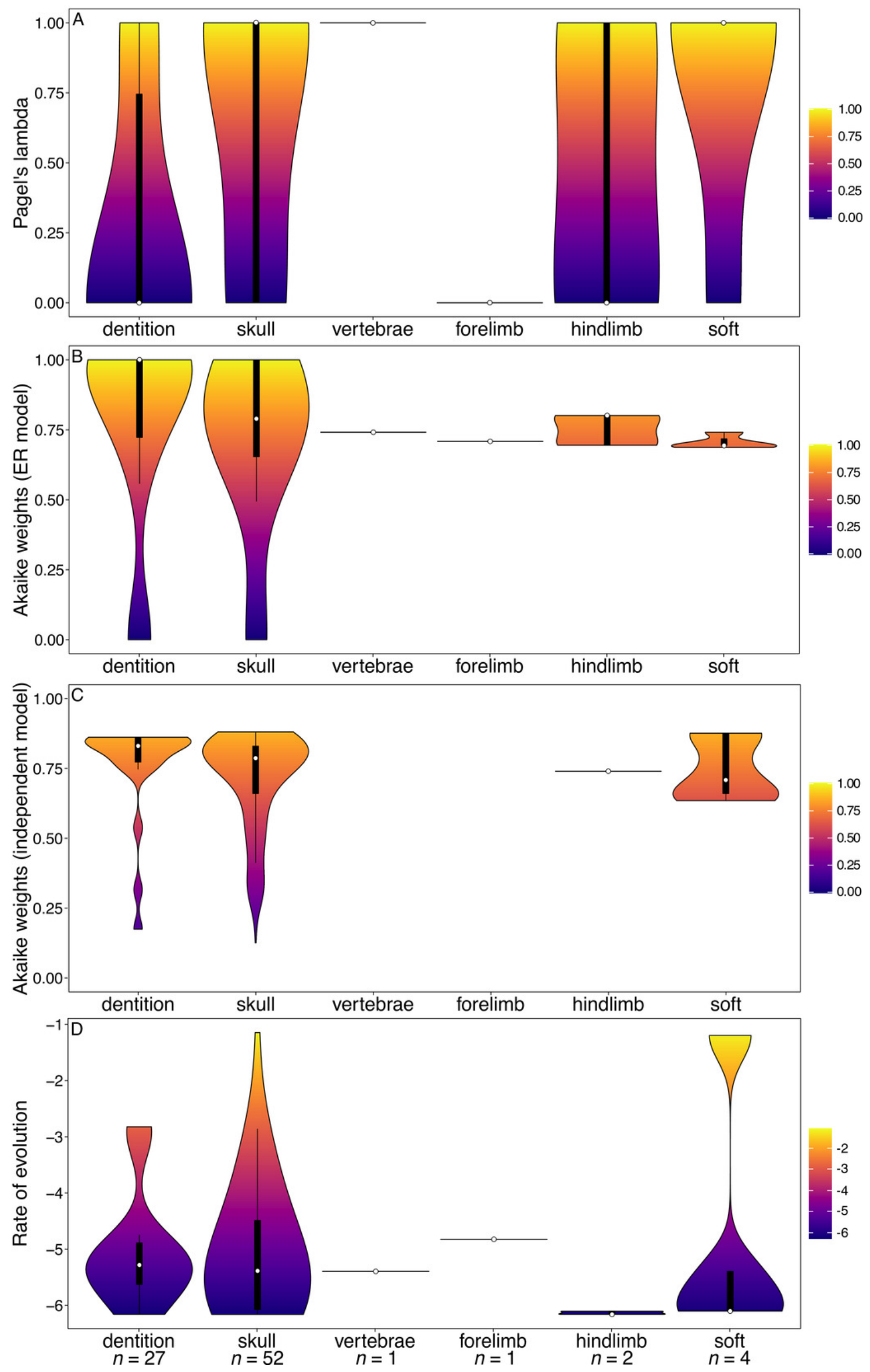

Peer] reviewing PDF | (2019:09:41216:2:0:NEW 29 Jan 2020) 


\section{Figure 4}

Figure 4: Violin plots illustrating results from the Ni et al. (2013) matrix (Primates)

A) Pagel's lambda values (phylogenetic signal) of each character. B) Akaike weights support for the ER model of evolution of each character. C) Akaike weights support for the independent model of evolution of all pairwise comparisons of characters in each partition. D) Rates of character evolution of each character ( $\log 10$ transformed). The number of characters in each partition can be found at the base of the figure $(n=\mathrm{X})$ 

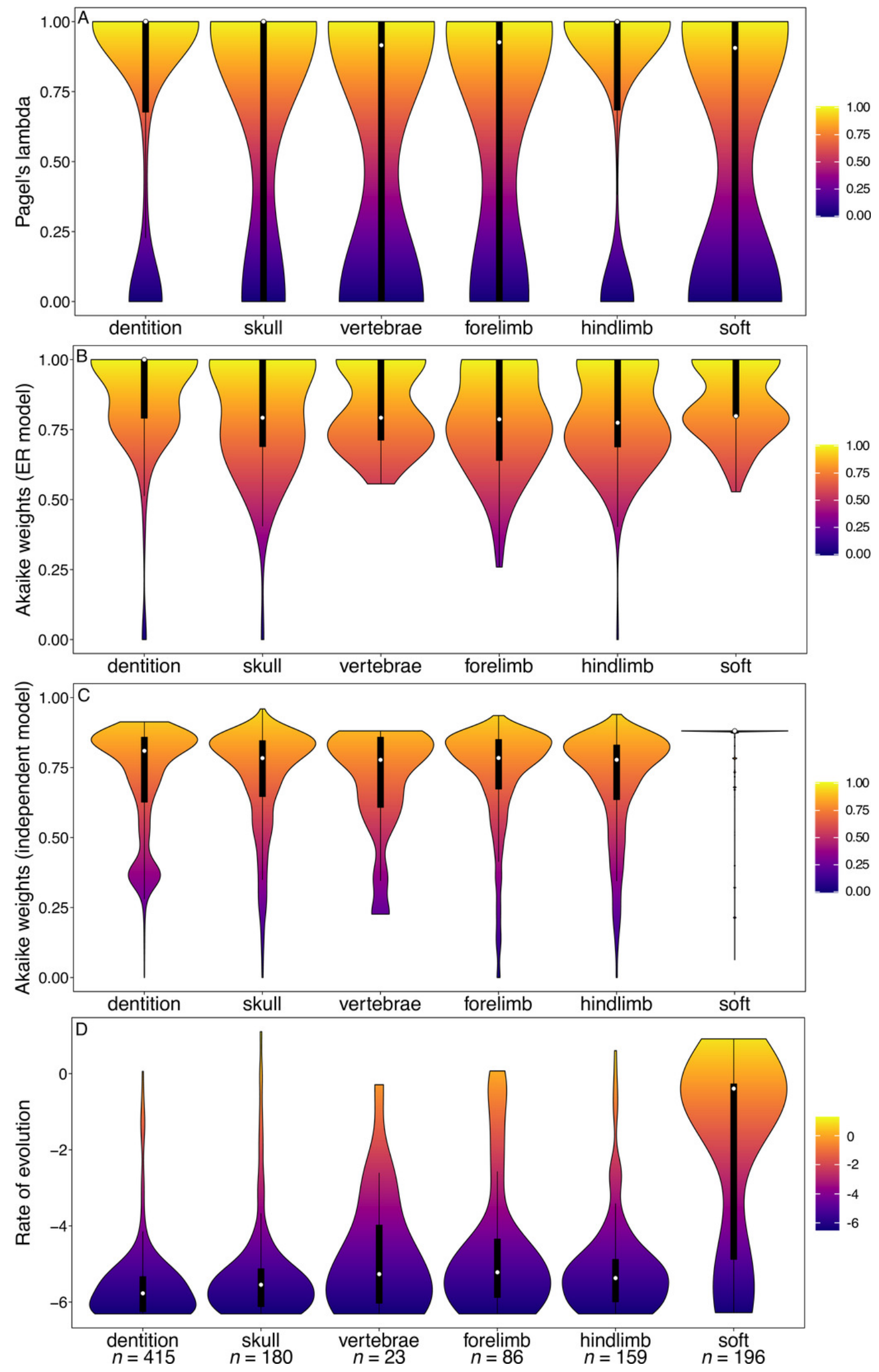

Peer) reviewing PDF | (2019:09:41216:2:0:NEW 29 Jan 2020) 


\section{Figure 5}

Figure 5: Violin plots illustrating results from the Beck (2017) matrix (Marsupialia).

A) Pagel's lambda values (phylogenetic signal) of each character. B) Akaike weights support for the ER model of evolution of each character. C) Akaike weights support for the independent model of evolution of all pairwise comparisons of characters in each partition. D) Rates of character evolution of each character ( $\log 10$ transformed). The number of characters in each partition can be found at the base of the figure $(n=\mathrm{X})$ 

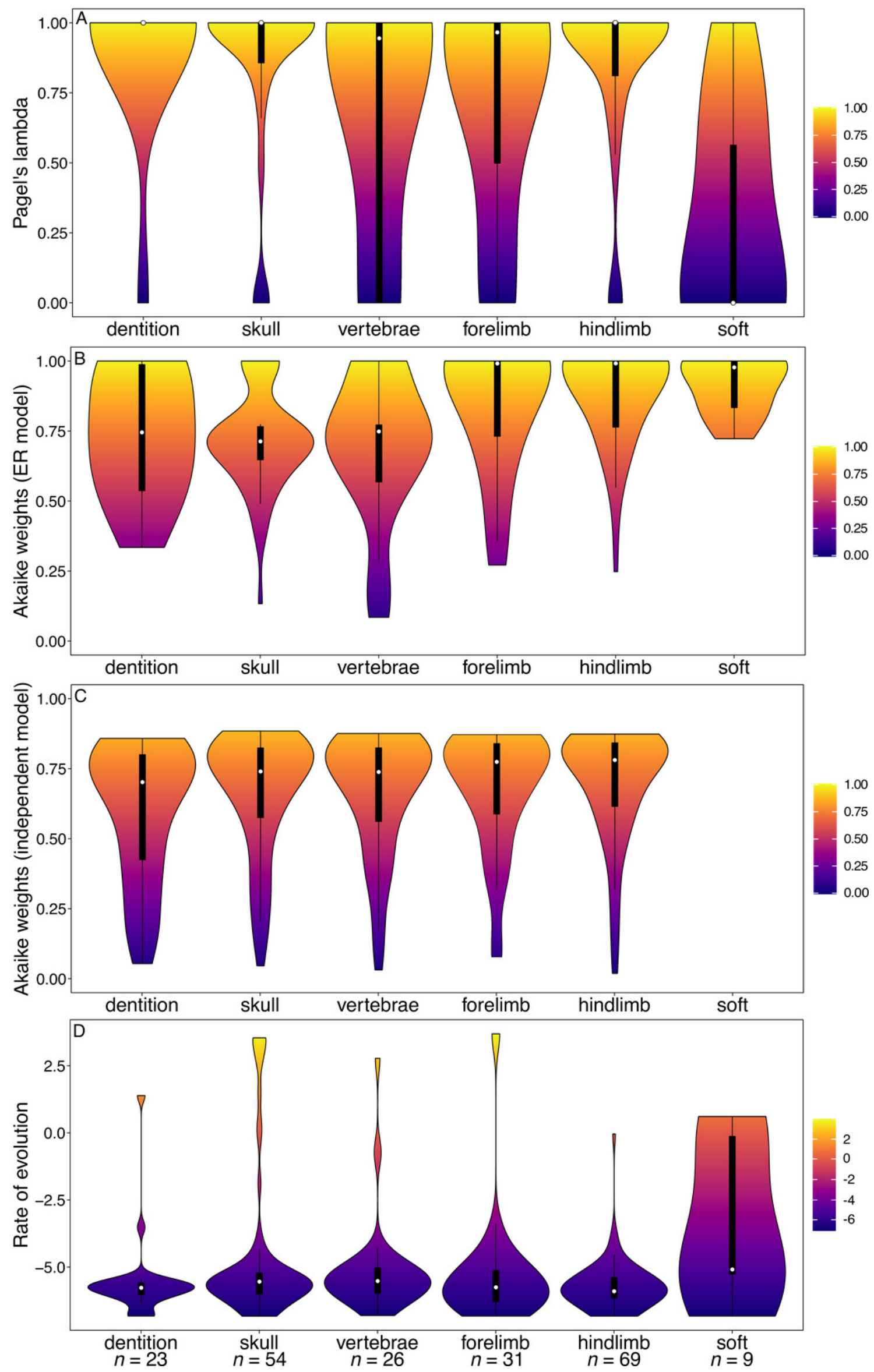

Peer) reviewing PDF | (2019:09:41216:2:0:NEW 29 Jan 2020) 


\section{Table $\mathbf{1}$ (on next page)}

Table 1. Results of GLS analyses of Pagel's $\lambda$ (phylogenetic signal of character partitions) in mammals.

Rows coloured are those where the partition best fits the $\mathrm{H} 1$ model (partition has a different lambda value to all others); blue indicates lower phylogenetic signal. 
1 Table 1. Results of GLS analyses of Pagel's $\lambda$ (phylogenetic signal of character partitions) in

2 mammals. Rows coloured are those where the partition best fits the H1 model (partition has a

3 different lambda value to all others); blue indicates lower phylogenetic signal.

\begin{tabular}{|c|c|c|c|c|c|c|c|c|}
\hline Partition & $\begin{array}{c}\text { Median } \\
\lambda\end{array}$ & $\begin{array}{c}\text { GLS } \\
\text { Coefficient }\end{array}$ & $\begin{array}{c}\operatorname{lnL} \\
(\text { null })\end{array}$ & $\begin{array}{c}\operatorname{lnL} \\
(\mathrm{H} 1)\end{array}$ & $\begin{array}{c}\text { AIC } \\
(\text { null })\end{array}$ & $\begin{array}{c}\text { AIC } \\
(\mathrm{H} 1)\end{array}$ & $\begin{array}{c}\text { Akaike } \\
\text { weights } \\
(\text { null })\end{array}$ & $\begin{array}{c}\text { Akaike } \\
\text { weights } \\
(\mathrm{H} 1)\end{array}$ \\
\hline Teeth & 1 & -0.15 & -103.02 & -102.6 & 210.0 & 211.1 & 0.63 & 0.37 \\
\hline Skull & 1 & -0.11 & -103.02 & -101.4 & 210.0 & 208.8 & 0.35 & 0.65 \\
\hline Vertebrae & 1 & 0.15 & -103.02 & -103.5 & 210.0 & 212.9 & 0.81 & 0.19 \\
\hline Forelimb & 1 & 0.16 & -103.02 & -101.3 & 210.0 & 208.5 & 0.32 & 0.68 \\
\hline Hindlimb & 1 & 0.07 & -103.02 & -103.8 & 210.0 & 213.6 & 0.86 & 0.14 \\
\hline Soft tissue & 1 & 0.15 & -103.02 & -103.4 & 210.0 & 2129 & 0.81 & 0.19 \\
\hline
\end{tabular}

4

5 


\section{Table 2 (on next page)}

Table 2. Results of GLS analyses of Akaike weight support for the equal rates (ER) model of character evolution in mammals. 
1 Table 2. Results of GLS analyses of Akaike weight support for the equal rates (ER) model of 2 character evolution in mammals.

\begin{tabular}{|c|c|c|c|c|c|c|c|c|}
\hline Partition & $\begin{array}{c}\text { Median } \\
\text { weight }\end{array}$ & $\begin{array}{c}\text { GLS } \\
\text { Coefficient }\end{array}$ & $\begin{array}{c}\operatorname{lnL} \\
\text { (null) }\end{array}$ & $\begin{array}{c}\operatorname{lnL} \\
(\mathrm{H} 1)\end{array}$ & $\begin{array}{c}\text { AIC } \\
\text { (null) }\end{array}$ & $\begin{array}{c}\text { AIC } \\
(\mathrm{H} 1)\end{array}$ & $\begin{array}{c}\text { Akaike } \\
\text { weights } \\
\text { (null) }\end{array}$ & $\begin{array}{c}\text { Akaike } \\
\text { weights } \\
\text { (H1) }\end{array}$ \\
\hline Teeth & 0.78 & 0.02 & 26.77 & 24.75 & -49.55 & -43.50 & 0.95 & 0.05 \\
\hline Skull & 0.71 & 0.02 & 26.77 & 24.45 & -49.55 & -42.89 & 0.97 & 0.03 \\
\hline Vertebrae & 0.62 & -0.13 & 26.77 & 26.60 & -49.55 & -47.19 & 0.76 & 0.24 \\
\hline Forelimb & 0.62 & -0.09 & 26.77 & 26.91 & -49.55 & -47.82 & 0.70 & 0.30 \\
\hline Hindlimb & 0.72 & 0.04 & 26.77 & 24.85 & -49.55 & -43.70 & 0.95 & 0.05 \\
\hline $\begin{array}{c}\text { Soft } \\
\text { tissue }\end{array}$ & 0.67 & 0.05 & 26.77 & 25.69 & -49.55 & -45.38 & 0.89 & 0.11 \\
\hline
\end{tabular}

3 


\section{Table 3 (on next page)}

Table 3. Results of GLS analyses of Akaike weight support for the independent model of character evolution in mammals.

Rows coloured are those where the partition best fits the $\mathrm{H} 1$ model (partition has a different rate value to all others); blue indicates lower Akaike weights, red indicates higher. 
1 Table 3. Results of GLS analyses of Akaike weight support for the independent model of

2 character evolution in mammals. Rows coloured are those where the partition best fits the H1

3 model (partition has a different rate value to all others); blue indicates lower Akaike weights, red

4 indicates higher.

\begin{tabular}{|c|c|c|c|c|c|c|c|c|}
\hline Partition & $\begin{array}{c}\text { Median } \\
\text { weight }\end{array}$ & $\begin{array}{c}\text { GLS } \\
\text { Coefficient }\end{array}$ & $\begin{array}{c}\operatorname{lnL} \\
\text { (null) }\end{array}$ & $\begin{array}{c}\operatorname{lnL} \\
(\mathrm{H} 1)\end{array}$ & $\begin{array}{c}\text { AIC } \\
\text { (null) }\end{array}$ & $\begin{array}{c}\text { AIC } \\
\text { (H1) }\end{array}$ & $\begin{array}{c}\text { Akaike } \\
\text { weights } \\
\text { (H0) }\end{array}$ & $\begin{array}{c}\text { Akaike } \\
\text { weights } \\
\text { (H1) }\end{array}$ \\
\hline Teeth & 0.69 & 0.012 & 1043.0 & 1040.5 & -2082 & -2075 & 0.97 & 0.10 \\
\hline Skull & 0.73 & 0.013 & 1043.0 & 1040.8 & -2082 & -2076 & 0.76 & 0.22 \\
\hline Vertebrae & 0.50 & -0.034 & 1043.0 & 1041.1 & -2082 & -2076 & 0.95 & 0.04 \\
\hline Forelimb & 0.57 & -0.077 & 1043.0 & 1073.1 & -2082 & -2140 & $\sim 0$ & $\sim 1$ \\
\hline Hindlimb & 0.75 & 0.039 & 1043.0 & 1050.7 & -2082 & -2095 & 0.001 & 0.999 \\
\hline $\begin{array}{c}\text { Soft } \\
\text { tissue }\end{array}$ & 0.76 & 0.100 & 1043.0 & 1042.4 & -2082 & -2079 & 0.83 & 0.16 \\
\hline
\end{tabular}

5 


\section{Table 4 (on next page)}

Table 4. Results of GLS analyses of rates of character evolution in mammals.

Rows coloured are those where the partition best fits the $\mathrm{H} 1$ model (partition has a different rate value to all others); red indicates higher rate. 
1 Table 4. Results of GLS analyses of rates of character evolution in mammals. Rows coloured are

2 those where the partition best fits the $\mathrm{H} 1$ model (partition has a different rate value to all others);

3 red indicates higher rate.

\begin{tabular}{|c|c|c|c|c|c|c|c|c|}
\hline Partition & $\begin{array}{c}\text { Median } \\
\text { rate }\end{array}$ & $\begin{array}{c}\text { GLS } \\
\text { Coefficient }\end{array}$ & $\begin{array}{c}\operatorname{lnL} \\
(\text { null })\end{array}$ & $\begin{array}{c}\operatorname{lnL} \\
(\mathrm{H} 1)\end{array}$ & $\begin{array}{c}\text { AIC } \\
\text { (null) }\end{array}$ & $\begin{array}{c}\text { AIC } \\
(\mathrm{H} 1)\end{array}$ & $\begin{array}{c}\text { Akaike } \\
\text { weights } \\
\text { (null) }\end{array}$ & $\begin{array}{c}\text { Akaike } \\
\text { weights } \\
(\mathrm{H} 1)\end{array}$ \\
\hline Teeth & 0.0016 & 0.29 & -53.01 & -43.63 & 110.03 & 93.27 & 0.0002 & 0.99 \\
\hline Skull & 0.0010 & 0.02 & -53.01 & -55.19 & 110.03 & 116.38 & 0.96 & 0.04 \\
\hline Vertebrae & 0.0006 & -0.20 & -53.01 & -52.59 & 110.03 & 111.19 & 0.64 & 0.36 \\
\hline Forelimb & 0.0006 & -0.08 & -53.01 & -53.76 & 110.03 & 113.52 & 0.85 & 0.15 \\
\hline Hindlimb & 0.0007 & -0.07 & -53.01 & -53.85 & 110.03 & 113.70 & 0.96 & 0.04 \\
\hline $\begin{array}{c}\text { Soft } \\
\text { tissue }\end{array}$ & 0.0006 & -0.21 & -53.01 & -53.12 & 110.03 & 112.24 & 0.75 & 0.25 \\
\hline
\end{tabular}

4 


\section{Table 5 (on next page)}

Table 5. Results of Kendal's tau correlation tests between rates of evolution and support for the equal rates model, and phylogenetic signal 
1 Table 5. Results of Kendal's tau correlation tests between rates of evolution and support for the

2 equal rates model, and phylogenetic signal

\begin{tabular}{|l|l|l|}
\hline & $\begin{array}{l}\text { Pagel's lambda vs Rates of } \\
\text { character evolution }\end{array}$ & $\begin{array}{l}\text { Pagel's lambda vs Akaike } \\
\text { weight support for ER model of } \\
\text { character evolution }\end{array}$ \\
\hline Total mammal dataset & $-0.22\left(\mathrm{p}=3.67 \times 10^{-6}\right)$ & $-0.050(\mathrm{p}=0.2996)$ \\
\hline Cetartiodactyl dataset & $-0.24\left(\mathrm{p}=3.49 \times 10^{-10}\right)$ & $0.15\left(\mathrm{p}=1.05 \times 10^{-4}\right)$ \\
\hline Carnivoran dataset & $-0.1(\mathrm{p}=0.4435)$ & $-0.04(\mathrm{p}=0.5701)$ \\
\hline Primate Dataset & $-0.22\left(\mathrm{p}=<2.2 \times 10^{-16}\right)$ & $-0.012(\mathrm{p}=0.56)$ \\
\hline Marsupial dataset & $-0.22\left(\mathrm{p}=2 \times 10^{-5}\right)$ & $0.11(\mathrm{p}=0.025)$ \\
\hline
\end{tabular}

3 


\section{Table 6(on next page)}

Table 6. Results of Kendal's tau correlation tests between number of characters in the partitions and phylogenetic signal, support for the equal rates and independent models, and rates of evolution. 
1 Table 6. Results of Kendal's tau correlation tests between number of characters in the partitions

2 and phylogenetic signal, support for the equal rates and independent models, and rates of

3 evolution.

\begin{tabular}{|l|l|l|}
\hline Correlation test & Kendall's tau & P value \\
\hline $\begin{array}{l}\text { Number of characters in dataset partition } \sim \text { Median Pagel's } \\
\text { lambda }\end{array}$ & -0.009 & 0.95 \\
\hline $\begin{array}{l}\text { Number of characters in dataset partition } \sim \text { Median Akaike } \\
\text { weights (ER model) }\end{array}$ & 0.31 & 0.10 \\
\hline $\begin{array}{l}\text { Number of characters in dataset partition } \sim \text { Median Akaike } \\
\text { weights (independent model) }\end{array}$ & 0.26 & 0.17 \\
\hline Number of characters in dataset partition $\sim$ Median rate & 0.28 & 0.12 \\
\hline
\end{tabular}

4 